travailler ensemble des pédiatres africains. Le contenu s'est voulu à la fois pratique et adapté à des particularités locales et à des ressources limitées. Toutefois, le plus grand défi dans le continent africain serait de fournir aux enfants atteints de maladie rénale l'accès à une prise en charge spécialisée par des néphrologues pédiatriques ayant une bonne formation ainsi que la possibilité d'avoir un accès abordable aux techniques modernes, aux médicaments efficaces et à des stratégies de prévention. Notre réseau est un bon point de départ puisque la majorité des pays africains francophones n'ont jamais été impliqué dans une activité de NP et connaissent de nombreuses difficultés dans le domaine de la santé. Un travail local, le renforcement de la collaboration régionale et internationale sont de nature à faire évoluer cette discipline dans nos pays.

Déclaration de liens d'intérêts L'auteur n'a pas précisé ses éventuels liens d'intérêts.

http://dx.doi.org/10.1016/j.arcped.2016.03.044

\section{CO 25}

Profil neuropsychologique et neuroanatomique chez 17 patients CrossMark français présentant une cystinose

A. Curie ${ }^{1,2,3}$, N. Touil 3 , S. Gaillard ${ }^{3}$, F. Cotton ${ }^{4}$,

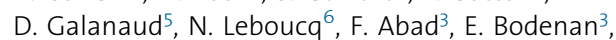

G. Deschênes ${ }^{7}$, D. Morin ${ }^{8}$, B. Kassai ${ }^{3}$, A. Bertholet-

Thomas $^{9}$, P. Cochat ${ }^{9, *}$

'Service de neuropédiatrie, hôpital Femme-Mère-Enfant, hospices civils de Lyon, Bron, France

${ }^{2}$ CNRS UMR 5304, L2C2, institut des sciences cognitives, Bron, France

${ }^{3}$ EPICIME-CIC 1407/Inserm, UMR5558, université de Lyon, hospices civils de Lyon, Bron, France

${ }^{4}$ Service de radiologie, centre hospitalier Lyon-Sud, hospices civils de Lyon, Pierre-Bénite, France

${ }^{5}$ Service de neuroradiologie, hôpital Pitié-Salpêtrière, AP-HP, Paris,

France

${ }^{6}$ Service de neuroradiologie, centre hospitalier universitaire de

Montpellier, Montpellier, France

${ }^{7}$ Service de néphropédiatrie, hôpital Robert-Debré, AP-HP, Paris,

France

${ }^{8}$ Service de néphropédiatrie, centre hospitalier universitaire de Montpellier, Montpellier, France

${ }^{9}$ Centre de référence des maladies rénales rares-néphrogones, filière ORKiD, Bron, France

${ }^{*}$ Auteur correspondant.

Adresse e-mail : pierre.cochat@chuyon.fr (P. Cochat)

Introduction La cystinose est une maladie lysosomale héréditaire rare, liée au déficit du transporteur de la cystine conduisant à une accumulation progressive de cystine dans les tissus. La tubulopathie proximale est l'un des premiers signes, évoluant vers l'insuffisance rénale terminale vers l'âge de 12 à 16 ans. D'autres atteintes apparaissent plus tardivement : endocriniennes, neurologiques et une myopathie. La cystéamine donnée précocement permet de retarder ces complications, allongeant l'espérance de vie. L'atteinte neurologique plus tardive demeure mal connue et évaluée. L'objectif de cette étude est de mieux comprendre le profil neuropsychologique et neuroanatomique lié à la cystinose.

Matériel et méthodes Dix-sept patients âgés en moyenne de 17,6 ans [5,4$33,3]$ et présentant une cystinose ont été inclus dans l'étude. Une évaluation du fonctionnement intellectuel (échelle de Wechsler adaptée à l'âge), de la mémoire (Children Memory Scale et Wechsler Memory Scale), et des capacités visuo-spatiales (figures de Rey) et visuo-perceptuelles ont été réalisées. Seize patients sur 17 ont effectué une IRM cérébrale $3 \mathrm{~T}$ (comprenant 3D T1 haute résolution, FLAIR, DTI, et spectroscopie).

Résultats L'efficience intellectuelle des patients présentant une cystinose est normale (QI total moyen $=100)$. Mais l'Indice de Raisonnement Perceptif (moyenne $=87,[63-109])$ est significativement plus bas que l'Indice de
Compréhension Verbale (moyenne $=100,[59-138], p=0,003$ ). L'évaluation des capacités mnésiques de ces patients révèle des capacités dans la norme, sans dissociation entre mémoire visuelle et verbale. Par contre, il existe une altération significative de la mémoire de travail par rapport aux capacités mnésiques générales $(p=0,003)$. L'évaluation de leurs capacités visuospatiales montre un score en copie et en rappel pour la figure de Rey inférieur au $50^{\mathrm{e}}$ percentile dans plus de $70 \%$ des cas. L'IRM révèle une atrophie corticosous-corticale à prédominance pariéto-occipitale.

Conclusion Les patients ayant une cystinose ont un profil neuropsychologique et neuroanatomique spécifique, dont la meilleure connaissance devrait améliorer leur prise en charge.

Déclaration de liens d'intérêts Les auteurs n'ont pas précisé leurs éventuels liens d'intérêts.

http://dx.doi.org/10.1016/j.arcped.2016.03.045

\section{CO 26}

Oral treatment of febrile urinary tract

infection in children: Feasibility and

follow-up

A. Dachy*, S. Eiras Da Silva, A. Thelen, L. Collard,

M.S. Ghuysen, J. Lombet, J. Maquet, P. Philippet

Pediatric department, CHC clinique de l'Espérance, Belgique

*Corresponding author.

E-mail address: angelique.dachy@gmail.com (A. Dachy)

Objective To assess the feasibility of oral treatment protocol with available oral antibiotics in Belgium in children admitted in a emergency unit in a single center for a febrile urinary tract infection.

Methods Participants/design - retrospective analysis of the outcomes of 316 children, aged 15 days to 15 years old presenting for a febrile UTI (positive dipstick for leucocyte esterase and/or nitrite + positive culture $(>1$ bacteria/ $\mu \mathrm{L},>50 \times 10^{3}$ and $>100 \times 10^{3}$ for suprapubic aspiration, bladder catheterization and mi-stream samples, respectively) from 1/01/2012 to $04 /$ 05/2015 in the pediatric emergency department of La clinique de l'Espérance. Methods - Children $\geq 3$ months with a first episode of febrile UTI were eligible for the oral treatment if there was no vomiting for $24 \mathrm{~h}$. Exclusion criteria were antibiotic therapy within the last 2 weeks, urinary tract surgery within the last month, history of dilated uropathy, abnormal antenatal ultrasound, renal transplantation, intermittent catheterization and parental inability to understand the treatment and the follow-up. The first dose was administered in the department. Children not fulfilling these criteria were hospitalized to receive IV antibiotic therapy and data were collected in order to compare epidemiology. The parents were called within the $72 \mathrm{~h}$ after admission to control time to apyrexia, vomiting, compliance and bacterial sensitivity to the given antibiotic. Primary outcome was to confirm the feasibility of oral treatment with the available antibiotics in Belgium where oral third generation cephalosporins are not available. Secondary outcome was the comparison of the sensitivity of bacteria identified in first episodes of UTI and in children with recurrent episode of UTI.

Results One hundred and fifty five $(52 \%)$ could be treated orally, 91 with amoxycillin-clavulanate, 71 with cefuroxime-axetil, 3 with TMP-SMX. The main reasons of ineligibility to the oral treatment were age $<3$ months $(36 \%)$, recurrent UTI (23\%), dilated uropathy (15\%) and vomiting within $24 \mathrm{~h}(13 \%)$. Oral treatment failure, i.e. hospitalisation, occurred in $17 \%$ of patients, mainly for vomiting $(11 \%)$ or persisting fever $(3 \%)$. The incidence of vomiting was similar between the children treated by amoxycillin-clavulanate and cefuroxime-axetil.

Discussion-conclusion Treatment with oral antibiotic can be implemented in the emergency department in a selected population providing a strict follow-up to assess parent's ability to administer effectively the treatment and their possibilities of representing the child in case of failure. Oral treatment with amoxycilline-clavulanate or cefuroxime-axetil is a safe option for a first episode of UTI in children $\geq 3$ months in our region. Thirteen percent of these children will experience vomiting which will hinder appropriate administration of the 
treatment. Oral cefuroxime-axetil is an option for children with recurrent episode of UTI as the causative bacteria was sensitive to this antibiotic in $98 \%$. Disclosure of interest The authors have not supplied their declaration of competing interest.

http://dx.doi.org/10.1016/j.arcped.2016.03.046

\section{CO 27}

La greffe rénale DVA-ABO incompatible

est une solution chez des enfants

hyperimmunisés sans accès à la greffe

F. Garaix ${ }^{1, *}$, C. Rousset-Rouviere', M. Cailliez',

D. Bruno', A. Basire', I. Dettori³, G. Hery', L. Daniel²,

M. Tsimarartos ${ }^{1}$

${ }^{1}$ Service de pédiatrie multidisciplinaire Timone, unité de néphrologie pédiatrique et transplantation, CHU Timone-Enfants, 13005 Marseille,

France

${ }^{2}$ Service d'anatomopathologie, CHU Timone, 13005 Marseille, France

${ }^{3}$ EFS Baille, 13005 Marseille, France

${ }^{4}$ Service de chirurgie infantile, viscérale et plastie, CHU Timone-

Enfants, 13005 Marseille, France

${ }^{*}$ Auteur correspondant.

Adresse e-mail : florentine.garaix@apm.fr (F. Garaix)

Les patients pédiatriques hyperimmunisés contre les antigènes HLA ont un accès extrêmement limité à la transplantation rénale. Le recours à une greffe $\mathrm{ABO}$ incompatible en cas de proposition de donneur vivant HLA compatible peut être une solution pour ces patients. Nous rapportons le cas d'une jeune fille greffée à 2 ans et demi pour IRCT (hypoplasie rénale), avec retour en dialyse 11 ans après. À l'inscription pour une seconde greffe, cette jeune fille avait un FAG à 0 et un taux de greffons incompatibles à $95 \%$ du à un large profil d'immunisation anti-HLA classe I et II. La recherche de DVA dans la famille a permis d'identifier son père comme étant le seul donneur sans DSA mais $\mathrm{ABO}$ incompatible (receveur $\mathrm{O}$ et donneur $\mathrm{A}$ ). Les autres donneurs proposés présentaient des DSA cytotoxiques ne permettant pas d'envisager une désimmunisation HLA. Après deux ans d'attente, la décision de proposer une greffe ABO incompatible a été prise. Les titres initiaux d'anti-A étaient de $1 / 128(\operatorname{Ig} M)$ (technique saline ne tube) et $1 / 64$ (IgG)(support filtration AGH), avec présence d'anticorps immuns. La préparation à la greffe (18 séances d'IA, 8 EP, Tacrolimus, Cellcept et corticoïdes et 2 injections de rituximab) a permis d'atteindre les titres cibles (1/8). La transplantation s'est déroulée sans complication notamment hémorragique. En post-greffe la créatinine s'est stabilisée à $90 \mu \mathrm{mol} / \mathrm{L}$. La réascension rapide des titres d'anti-A ont motivé $4 \mathrm{EP}$ post-greffe. Les biopsies réalisées à M1 et M3 ne montrent aucun signe de rejet humoral, et comme attendu, un marquage $\mathrm{C} 4 \mathrm{~d}$ diffus. Actuellement, à un an post-greffe, les titres sont stables à $1 / 64(\operatorname{IgM})$ et $1 / 16(\operatorname{IgG})$ et la créatinine est à $95 \mu \mathrm{mol} / \mathrm{L}$ sous tacrolimus, MMF et corticoïdes. La greffe $\mathrm{ABO}$ incompatible avec DVA permet d'offrir un accès à la transplantation rénale à des enfants hyperimmunisés en l'absence de DVA HLA compatible, avec la perspective d'une meilleure survie au long court que la greffe HLA incompatible.

Déclaration de liens d'intérêts Les auteurs n'ont pas précisé leurs éventuels liens d'intérêts.

http://dx.doi.org/10.1016/j.arcped.2016.03.047

\section{CO 28}

Étude de l'épidémiologie et de la morbidité liées à l'EBV chez les greffés

rénaux d'âge pédiatrique

A. Laurent', J. Bacchetta', B. Kassai Koupai², P. Roy³,

P. Cochat, ${ }^{1, *}$

${ }^{1}$ Service de néphrologie, rhumatologie et dermatologie pédiatriques, hôpital Femme-Mère-Enfant, hospices civils de Lyon, France
${ }^{2}$ Centre d'investigation clinique de Lyon, groupement hospitalier Est-hôpital Mère-Enfant-EPICIME, France

${ }^{3}$ Laboratoire de biostatistique, hospices civils de Lyon, France

${ }^{*}$ Auteur correspondant.

Adresse e-mail : pierre.cochat@chuyon.fr (P. Cochat)

Introduction Les patients transplantés rénaux à l'âge pédiatrique ont plus de risque de contracter une primo-infection au virus d'Epstein-Barr (EBV) puisqu'ils sont souvent séronégatifs pour l'EBV à la transplantation. Une forte réplication d'ADN est reconnue comme un facteur de risque de développer une maladie lymphoproliférative post-transplantation (PTLD). Cependant, bien que la majorité des patients avec un PTLD possède une charge virale EBV élevée, certains patients développent un PTLD malgré une charge virale faible. L'objectif de cette étude est d'identifier les facteurs de risque (FDR) de réactions sévères à l'EBV.

Matériel et méthodes Cette étude monocentrique rétrospective concerne 117 enfants transplantés rénaux entre janvier 2003 et décembre 2012. Les infections sévères ont été arbitrairement définies comme la survenue d'un PTLD ou la présence d'une charge virale $>10000$ copies $/ \mathrm{mL}$. Un modèle de survie à taux proportionnel de Cox a été réalisé pour identifier les facteurs de risques de réaction sévère avec calcul du taux relatif (TR).

Résultats Sur un total de 117 patients (46 patients séronégatifs pour l'EBV à la greffe, âge moyen à la greffe $9,7 \pm 5,27$ ans), 54 patients ont eu une PCR positive ( $>500$ copies $/ \mathrm{mL})$ durant leur suivi ; il s'agissait d'une primoinfection dans 22 cas ; 24 patients ont présenté une réaction sévère et 4 un PTLD. Trois FDR d'infection sévère sont identifiés : un mismatch EBV à la greffe $(\mathrm{TR}=4,1, p<0,001)$, l'âge inférieur à 5 ans à la greffe $(\mathrm{TR}=4,13$, $p<0,001$ ), et la perte des anticorps anti-EBNA pendant le suivi (TR $=6,1$, $p=0,009)$.

Discussion En plus des FDR classiquement identifiés retrouvés dans cette étude (âge à la greffe, mismatch EBV), le monitorage des anticorps antiEBNA en post-greffe pourrait permettre de surveiller plus étroitement les enfants à risque d'infection sévère.

Déclaration de liens d'intérêts Les auteurs n'ont pas précisé leurs éventuels liens d'intérêts.

http://dx.doi.org/10.1016/j.arcped.2016.03.048

\section{CO 29}

Résultats de la transplantation rénale chez les jeunes enfants : une étude appariée sur 32 enfants de moins de trois ans

Y. Loiseau' ${ }^{1,2, *}$, J. Bacchetta ${ }^{1}$, P. Roy ${ }^{3}$, F. Nobili4,

F. Garaix', A. Laurent', P. Cochat'

${ }^{1}$ Néphrologie pédiatrique, hospices civils de Lyon, Bron, France

${ }^{2}$ Pédiatrie, hôpital Nord-Franche-Comté, Belfort, France

${ }^{3}$ Service de biostatistiques, hospices civils de Lyon, Lyon, France

${ }^{4}$ Néphrologie pédiatrique, CHRU Jean-Minjoz, Besançon, France

${ }^{*}$ Auteur correspondant.

Adresse e-mail : jorday25@hotmail.fr (Y. Loiseau)

Objectifs Malgré une amélioration considérable au cours des dernières années, la transplantation rénale avant l'âge de trois ans demeure un challenge.

Matériels et méthodes Nous avons étudié rétrospectivement le devenir de chaque enfant transplanté avant trois ans dans le centre de Lyon depuis 1987 $(n=32, \mathrm{Gl})$. Ces derniers ont été appariés sur le type de donneur et l'année de transplantation avec des enfants âgés de 3 à 13 ans $(n=32, \mathrm{G} 2)$ et 13 à 18 ans $(n=32, \mathrm{G} 3)$.

Résultats Aucune différence n'est apparue concernant le sexe, l'étiologie rénale initiale, le nombre d'enfants dialysés avant transplantation, le poids, la taille, la durée d'ischémie froide, les mismatches CMV et HLA, l'immunosuppression et la durée de suivi. Dans $\mathrm{Gl}$, la dialyse péritonéale $(p<0,001)$, 